\title{
TELMA: technology-enhanced learning environment for minimally invasive surgery
}

\author{
Patricia Sánchez-González, \\ Daniel Burgos, \\ Ignacio Oropesa, \\ Vicente Romero, \\ Antonio Albacete, \\ Luisa F. Sánchez-Peralta, \\ José F. Noguera, \\ Francisco M. Sánchez-Margallo, \\ Enrique J. Gómez,
}

Background: Cognitive skills training for minimally invasive surgery has traditionally relied upon diverse tools, such as seminars or lectures. Web technologies for e-learning have been adopted to provide ubiquitous training and serve as structured repositories for the vast amount of laparoscopic video sources available. However, these technologies fail to offer such features as formative and summative evaluation, guided learning, or collaborative interaction between users.

Methodology: The "TELMA" environment is presented as a new technology-enhanced learning platform that increases the user's experience using a four-pillared architecture: (1) an authoring tool for the creation of didactic contents; (2) a learning content and knowledge management system that incorporates a modular and scalable system to capture, catalogue, search, and retrieve multimedia content; (3) an evaluation module that provides learning feedback to users; and (4) a professional network for collaborative learning between users. Face validation of the environment and the authoring tool are presented. Results: Face validation of TELMA reveals the positive perception of surgeons regarding the implementation of TELMA and their willingness to use it as a cognitive skills training tool. Preliminary validation data also reflect the importance of providing an easy-to-use, functional authoring tool to create didactic content.

Conclusion: The TELMA environment is currently installed and used at the Jesús Usón Minimally Invasive Surgery Centre and several other Spanish hospitals. Face validation results ascertain the acceptance and usefulness of this new minimally invasive surgery training environment. 


\section{1. \\ Introduction}

One of the most important revolutions in clinical practice in the past few decades has been the introduction of minimally invasive surgery (MIS) techniques [1], which have spread among nearly all surgical specialties. MIS techniques have improved patient safety, reduced tissue damage, decreased morbidity, and shortened the duration of hospital stays for patients. However, MIS techniques imply a different and limited interaction paradigm compared with the traditional open approach $[1,2]$.

This paradigm shift has brought forth a necessary change in education programs for new surgeons: because of the reduced time available for education, traditional training based on Halsted's mentor-apprentice relationship [3] is no longer sufficiently efficient. Thus, it is gradually being replaced by new reproducible and flexible methods in which active learning is encouraged. Effective training and assessment of surgeons has become one of the major concerns of hospitals and clinics in recentyears, fuelled mostly by patients' and society's demands for safer surgeries and better-prepared physicians [2]. The development of standardized training processes is the key to success in the optimization of the trainee's learning curve, whereas proper accreditation relies on developing objective curricula in which personal bias can be reduced to a minimum.

There is a series of skills that a surgeon must master to achieve proficiency [4]. These skills can generally be classified into cognitive (acquisition of the necessary theoretical knowledge), motor (mastering of the surgical gestures and processes), and judgment skills (knowing what to do and how to react when faced with different scenarios) $[5,6]$. Cognitive knowledge can be obtained by different means, such as seminars and courses, or by employing multimedia didactic material in the form of books, CD-ROMs [7], and especially endoscopic videos, which are one of the most important sources of information available in this field [8].

The adoption of web technologies for cognitive online training can be a useful way of optimizing education programs, allowing for anytime/anywhere training [9]. The evolution of technology-enhanced learning (TEL) has led to the development of learning management systems, like .LRN and Moodle, which enable organizations to plan and track their learning needs; as well as learning content management systems $[10,11]$, which provide tools to deliver instructor-led synchronous and asynchronous online training, allowing content creation, reuse, search, delivery, and management. Combined with the strengths provided by laparoscopic videos [12], e-learning in surgery is an effective way of breaking time, space, and cost barriers by offering online or blended education alternatives that are potentially more viable and feasible than on-site courses.

Currently, there are several online surgical repositories available. Alternatives such as WebSurg [13], WebOp [14], and Surgytec [15], among others, represent different approaches and examples of enhanced medical learning environments that offer a web video repository. Adoption of these websites among the medical community is suffering unduly. There are several possible causes for this lack of adoption: (1) not all videos are properly documented and explained; (2) didactic content is not easily created because of the nonexistence of authoring tools; (3) user interaction with the content is at best reduced, limited to simple navigation between sequential content; (4) trainee evaluation is all but nonexistent; and (5) contact between users is rarely encouraged.

We present the system description and face validation of TELMA, a new TEL environment in which the didactic value of surgical videos is better exploited thanks to the possibility of editing and enrichment. This platform is envisaged as a new cognitive learning concept to create, share, and reuse scalable didactic content and to adapt the content to learners' individual needs according to validated pedagogical principles. User education is also boosted, allowing more active participation in the learning process, enabling objective surgical skills assessment, and encouraging collaboration with other surgeons and trainees with different surgical expertise.

\section{Methodology}

\subsection{Environment description}

The TELMA design has been performed based on a usercentered scheme. In a previous study, we reported results regarding users' needs and requirements to determine the functionalities and pedagogical principles to apply in the environment [16,17]. Questionnaire samples included different surgical profiles: expert teachers, expert surgeons, and novices. The major conclusion arrived at was that an e-learning cognitive environment would need to promote surgical videos as the core training element, making possible the objective evaluation of skills and the recommendation of content while incorporating social network services.

TELMA combines in a structured fashion the use of MIS didactic content and knowledge and learning resources. This is achieved through the design of (1) a multimedia didactic content authoring tool; (2) a learning content and knowledge management system (LCKMS), incorporating the MetaMender recommender system [17]; (3) an objective evaluation module; and (4) a surgical professional network for medical students and surgeons. These four elements make up the main logical pillars of the environment (Fig. 1).

\subsubsection{Multimedia didactic authoring tool}

The first logical pillar, the multimedia authoring tool, allows building of scalable didactic content from individual users' knowledge. Authoring tools are used to plan, develop, edit, publish, and maintain learning content. Moreover, software programs offer packages of resources that include the design of didactic materials, lectures ready to take to the classroom, training courses at different levels, and a pool of multimedia objects, among others (Learning Activity Management System).

The TELMA multimedia didactic authoring tool offers the creation of formal learning content, the efficient use of surgical videos, and the addition of multimedia objects to enhance surgical explanations. TELMA's authoring tool takes into consideration that creation is not simply a matter of digitizing traditional materials; it also involves new approaches to forming well-designed surgical educational resources. 
TELMA's MIS training environment

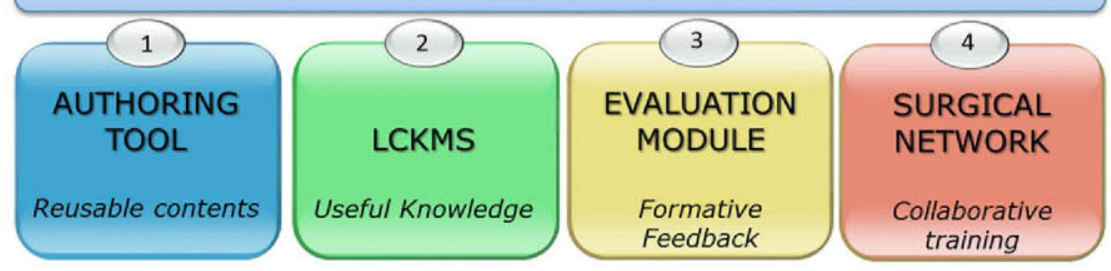

Fig. 1 - TELMA's logical pillars. Bioengineering and Telemedicine Centre (GBT).

The didactic tool allows the authoring of three different types of content in the learning environment: didactic units, case reports, and stand-alone videos. Thus, it allows the creation of complex formal content from several didactic objects, including new descriptive elements to improve the content's didactic value (Fig. 2). As video processing is needed to enhance information present in surgical videos, the TELMA authoring tool incorporates a surgical video editor especially designed for this purpose.

The video editor developed is designed with a multilayered structure to facilitate information addition or suppression. Its functionalities can be grouped into the following categories: archive operations (open, save, and close videos), image processing operations (e.g., segmentation and tracking of structures), enhancement operations (adding voice recordings and textual information), interactive operations (adding didactic exercises), interoperability operations (insertion and search of tags), content visualization operations (reproduction control of laparoscopic videos and tracking of regions of interest), and other miscellaneous operations (e.g., clipping, insertion of medical images, and insertion of documents).

Because of the importance of this tool, the user interface has a user-centered design according to usability and accessibility criteria that make it easier to use. Therefore, in its development, it was taken into consideration that, in this specific case, the average user is not used to employing this kind of software tool.

The TELMA multimedia didactic authoring tool is provided to users in two different formats: downloadable software for advanced video analysis and processing (object segmentation and tracking) and a simplified online version with simple editing functionalities. The TELMA offline authoring tool has been developed in $\mathrm{C}++$, using OpenCV library for the development of video processing algorithms. The online authoring version has been implemented in PHP.
2.1.2. Learning content and knowledge management system Users' knowledge management is achieved within the second pillar. The LCKMS provides adaptive learning to users based on their progress (formal learning) and behavior (informal learning) within their continuous formative path using the environment. Recommendations are given to users regarding the most suitable content, colleagues working in the same field, and interactions to perform, given their personal interests and progress in TELMA.

The TELMA environment includes a learning content and knowledge management system composed of a content manager, a knowledge management system, and an adaptation engine supporting a recommendation layer. In comparison with existing learning management systems and leaming content management systems, the LCKMS allows: (1) monitoring the activities of TELMA users; (2) interpreting these activities to update trainees' learning curves; (3) automatically inferring special user requirements; (4) acting upon the available knowledge; and (5) receiving personalized recommendations centered on formative paths.

2.1.2.1. Content management system. A content management system (CMS) is a modular and scalable system used to capture, catalogue, search, and retrieve multimedia content. The role of the CMS within TELMA is to manage video, audio, image, and document files. New content is uploaded by users from the authoring tool via an embedded file transfer protocol, where the system converts video files to the different resolutions and qualities required. All resolutions are perfectly synchronized though time codes, thus allowing choice of format at any time. Multilanguage documentation is handled via metadata fields generated in a relational data model through a properly predefined clinical thesaurus. In addition, TELMA allows users to detail the content to the level of thumbnails, where clips are documented and retrieved through the search process in the

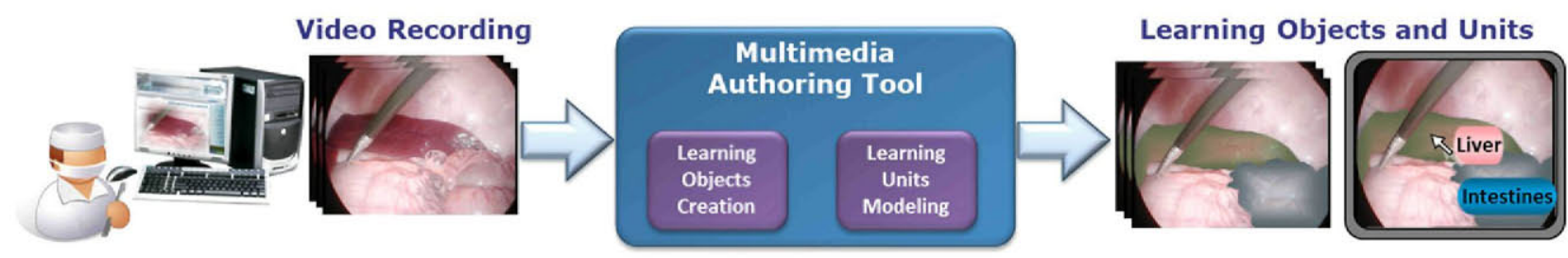

Fig. 2 - TELMA's multimedia authoring tool. Bioengineering and Telemedicine Centre (GBT). 
TELMA environment. Once the content is properly processed, it is published into the TELMA environment by means of web services, where information is presented according to users' requirements and recommendations.

The TELMA CMS runs on Windows XP and Windows 2003 Server Standard Edition (Microsoft). Applications are developed using PHP for the web environment and .Net for the Win32 environment. Zend (Zend Technologies, Germany) is used as the encryption and optimization technology.

2.1.2.2. Adaptation engine: recommendation layer. As e-learning aims at supporting learners in fulfilling specific training needs within a specific education context, a recommendation system is included in TELMA, named Meta-Mender, to provide personalized recommendations to users [17]. In this learning environment, adaptation is used to describe not only the adjustment of the interface but also the personal experience of learning through a specific user-adapted itinerary, with related content, interface, flow, evaluation, and other features. Thus, relevant feedback mechanisms for a dynamic update of the system based on learning object modifications and behavior adaptation are contemplated.

TELMA features an adaptation engine (Fig. 3) that considers initial users' data (e.g., profiles, preferences, and objectives), information regarding users' interactions, and performance. It provides personalized recommendations centered on formative paths. Likewise, the Meta-Mender recommendation system enables lifelong learning, where formative and evaluation stages follow each other continuously. In this manner, the system is able to track the learner's evolution and adapt accordingly.

TELMA's adaptation process can be described by three stages: (1) retrieving the information about the user; (2) processing the information to initialize and update a user model; and (3) using said user model to provide the adaptation through Meta-Mender.

The user model, closely related to the adaptation engine, is a dynamic entity within the environment that gathers users' skills and data. As preferences and requirements differ from each user and vary during the use of the TELMA environment, the model must change and adapt to them. The result is a model that stores all users' information, whether it be static (personal or professional data) or dynamic (interests, preferences, and concerns), and their associations.

A recommendation agent is also integrated into the TELMA learning environment. One of the TELMA requirements demands that users be able to receive cognitive learning guidance in locating and ranking contents. Thus, real-time personalized recommendation is achieved, providing users with didactic content suitable for their own learning requirements. To achieve this goal, a rule engine, DROOLS, has been used for determining the best recommendation at any given moment. Meta-Mender uses DROOLS to define meta-rules, which are complex rules that generate user-adapted rules. These are later employed to provide the final recommendations to users. Since user-adapted rules tend to remain the same during a session, it is possible to obtain them once and store them for further use. In this way it is possible to reduce the recommendation response time. Meta-Mender can provide several kinds of recommendations, such as which content one should study next or which users one should follow.

2.1.2.3. Knowledge management system. The knowledge management system (KMS) involves the creation of useful knowledge from information or data found in the available resources. The KMS monitors all interactions and processes them within the TELMA environment, allowing the creation, capture, storage, sharing, and distribution of information and turning it into knowledge that can then be shared by TELMA users. The KMS tracks the user's progress and profile, compares them with learning methodologies, and selects the appropriate adaptation models that are optimal for each particular user. By monitoring the user's learning process, the KMS is able to acquire the evolution of users' learning, so that training is adapted accordingly.

\subsubsection{Evaluation system}

Effective learning requires that objective, immediate feedback be given to the trainee in order to guide his or her progress. Ideally, this feedback must come in two forms: formative and summative [18-20]. Formative feedback will direct the trainees' progress during the training process, giving them useful information to immediately correct their mistakes. Summative feedback, on the other hand, will deal with the end result, where a final score is given with academic purposes.

In TELMA, both forms of feedback are addressed. Three different types of exercises are available in the authoring tool for the inclusion of evaluation units within the didactic content of the environment. Each exercise will belong to a different category, comprising "anatomical," "tools," "procedural," and "complications." Their inclusion within these resources allows

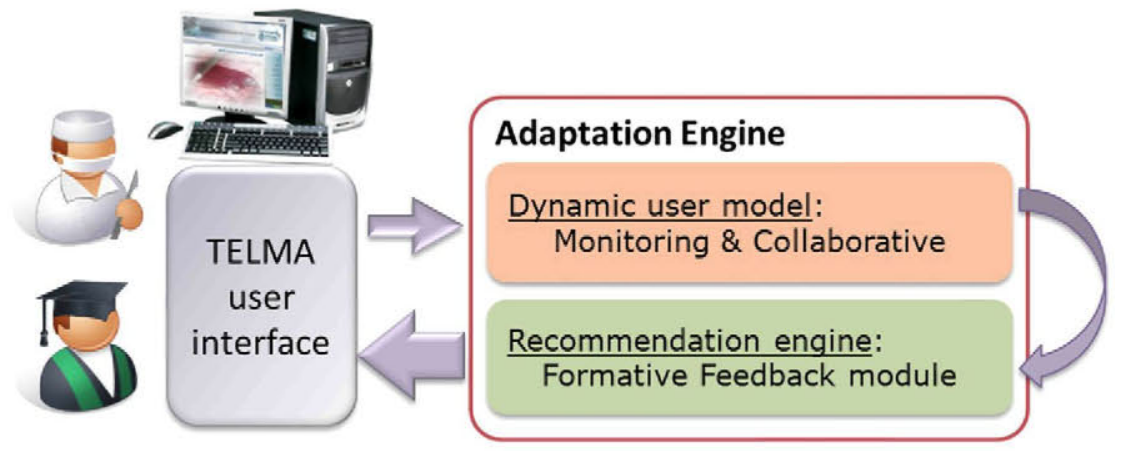

Fig. 3 - Adaptation engine subsystems. Bioengineering and Telemedicine Centre (GBT). 
for the creation of reports where, after completing an evaluation unit, information is given to trainees about their final score (summative) and their mistakes (formative). Information is presented in the form of text and graphic reports at any given time, which allow users to:

- visualize their learning curve, for the total of evaluation units performed or filtered by date and/or specialty

- look up any past evaluation units performed, by interacting with the learning curve (option is given for repeating them to improve scores)

- compare their grades for a given test with the average scores of the different user groups in TELMA.

- view their progress in terms of categories, comparing the number of correctly answered questions with the total performed.

To guide the academic path of the trainee in TELMA, scores are also assimilated into the KMS via the user model. The adaptation engine will use this information whenever recommendations are required by the environment for a given user. The information provided by the KMS will be able to suggest content to users, depending on their past performances and those of users of similar characteristics.

\subsubsection{Surgical professional collaborative network}

The final pillar in TELMA is the professional network, which allows for the creation of collaborative networks of students and professionals (informal learning is then enhanced continuously and in a form transparent to users regardless of their current activity). A growing body of research has demonstrated that professional networking is one of the central elements in collaborative learning environments [21-23]. Researchers have confirmed that these networks also play instrumental roles in learning environments as a conduit of resource and knowledge exchange [24] and as a source of support and socialization for distributed learners [23]. Thus, e-learning processes are more effective when trainee interaction is promoted, whether with the system itself or with other users.

TELMA's surgical professional network is a decentralized concept throughout the learning environment. Thus, informal learning is enhanced continuously, offered in a transparent form to users no matter their current activity within TELMA.

Within this surgical professional network, users of different expertise levels can communicate with each other, collaborate, and contribute their own medical experiences. The network supports synchronous and asynchronous communication between users within the learning environment to encourage user interaction. The informal learning processes are thus improved, as users share their medical knowledge and experiences through individual and group interactivity as well as useful information, doubts, and opinions among others. The creation of collaborative learning communities, which might enhance the sense of competency acquired by user participation, is promoted in this manner.

\subsection{Validation}

In order to determine the usefulness of the TELMA environment, face validation was carried out at the Jesús Usón
Table 1 - TELMA face validation tasks.

\begin{tabular}{|c|c|}
\hline Task performed & Task achievement \\
\hline Free navigation & - \\
\hline $\begin{array}{l}\text { Create account in TELMA } \\
\text { environment }\end{array}$ & $\begin{array}{l}\text { Confirmation of new account is } \\
\text { displayed }\end{array}$ \\
\hline $\begin{array}{l}\text { Didactic unit visualization and } \\
\text { evaluation performance }\end{array}$ & Evaluation results are displayed \\
\hline $\begin{array}{l}\text { Creation of a new post in the } \\
\text { professional network }\end{array}$ & $\begin{array}{l}\text { The post can be visualized in } \\
\text { TELMA }\end{array}$ \\
\hline $\begin{array}{l}\text { Latest news visualization and } \\
\text { following }\end{array}$ & $\begin{array}{l}\text { The user is following the news } \\
\text { content }\end{array}$ \\
\hline $\begin{array}{l}\text { Message sending and user } \\
\text { following }\end{array}$ & $\begin{array}{l}\text { The message has been sent and } \\
\text { the user is now being followed }\end{array}$ \\
\hline $\begin{array}{l}\text { Surgical video online } \\
\text { enhancement }\end{array}$ & $\begin{array}{l}\text { The enhanced video is visible in } \\
\text { TELMA }\end{array}$ \\
\hline Free navigation - user logged & - \\
\hline Session closing & The user is now logged out \\
\hline
\end{tabular}

Minimally Invasive Surgery Centre and several national hospitals. Five expert surgeons ( $>100$ interventions) and two trainees (with no laparoscopic experience) took part in this preliminary validation. They were requested to complete a set of tasks within the TELMA environment (Table 1) and were later allowed to surf without restriction in TELMA. Completion of any of the tasks was not forced upon the participants. Finally, all participants filled out a questionnaire comprising 38 yes/no, Likert $[25,26]$, and open questions in order to assess usability and content aspects. The Likert scale used was as follows: strongly agree (5), agree (4), not sure/undecided/ neutral (3), disagree (2), and strongly disagree (1). Because no

\section{Table 2 - Authoring tool face validation tasks.}

\begin{tabular}{|c|c|}
\hline Task performed & Task achievement \\
\hline \multicolumn{2}{|l|}{$\begin{array}{l}\text { Stand-alone video } \\
\text { enhancement }\end{array}$} \\
\hline $\begin{array}{l}\text { Import a video previously } \\
\text { enhanced }\end{array}$ & $\begin{array}{l}\text { The imported enhanced video } \\
\text { is visible in the authoring tool }\end{array}$ \\
\hline Save an image & $\begin{array}{l}\text { The image file is created in the } \\
\text { folder selected by the user }\end{array}$ \\
\hline Video cutting & $\begin{array}{l}\text { Video duration is adapted to the } \\
\text { selected frames }\end{array}$ \\
\hline Text introduction & $\begin{array}{l}\text { The text is introduced over the } \\
\text { video frames }\end{array}$ \\
\hline $\begin{array}{l}\text { Segmentation and tracking } \\
\text { from } 2 \mathrm{~s} \text { to } 1 \mathrm{~min} 14 \mathrm{~s}\end{array}$ & $\begin{array}{l}\text { A confirmation dialog is shown } \\
\text { and the mask is drawn over the } \\
\text { video }\end{array}$ \\
\hline Clip creation & $\begin{array}{l}\text { The created clip is visualized in } \\
\text { the authoring tool }\end{array}$ \\
\hline Metadata introduction & $\begin{array}{l}\text { Video tags are showed beside } \\
\text { the video in the authoring tool }\end{array}$ \\
\hline $\begin{array}{l}\text { Free navigation and use } \\
\text { Case report creation }\end{array}$ & - \\
\hline $\begin{array}{l}\text { Free navigation between sheets } \\
\text { Didactic unit development }\end{array}$ & - \\
\hline Free navigation between sheets & - \\
\hline
\end{tabular}

Bioengineering and Telemedicine Centre (GBT). 
standardized questionnaires were found completely suitable for our purpose, the questionnaire was ad hoc designed based on the Questionnaires for User Interaction Satisfaction (QUIS).

Additionally, a parallel face validation of the authoring tool was also carried out. The key core of the TELMA environment relies on the efficient use and creation of didactic content based on laparoscopic videos. Thus, one of the determining factors in the acceptance of the surgical e-learning environment is the efficient use of the authoring tool included. The validation process was carried out by five surgeons: four experts (more than 100 laparoscopic procedures performed) and one trainee with no laparoscopic experience. The methodology addressed in the validation study consisted of a test with two parts to it. In the first part, a technologist explained the aim of and the functionalities involved in the authoring tool. Afterwards, the surgeon performed a series of tasks (Table 2) with the authoring tool, under the supervision of the developer, for $5 \mathrm{~min}$. In the second part, supervision was avoided and surgeons were allowed to freely use the authoring tool, with no time constraints imposed. Finally, an interview was done and a QUIS questionnaire was completed to obtain information regarding operational, functional, and usability data. The questionnaire for validation processes was composed of Likert $[25,26]$ questions.

\section{Results}

\subsection{TELMA environment validation}

Results of the environment validation are presented in Figure 4 . Over $60 \%$ of participants answered positively to yes/ no questions. Additionally, average scores for usability, design, and content rate over 4 for Likert-type questions. Open questions highlight the need for optimizing the efficiency of the environment, improving the time needed for editing, and navigation through the environment.

\subsection{Multimedia authoring tool validation}

Results for the validation of the authoring tool are shown in Figure 5. Operation of the tool, video editor functionalities, ease of use and learning, and usability have been analyzed, showing good preliminary results (values over 3 ) in most of the issues studied. Results highlight the need for working on robustness and stability improvement, then achieving an easier and motivated use of the authoring tool. Regarding video editor functionalities, results show a good initial user acceptance. Although the most valuable functions are the cutting and clipping, organs' and tools' segmentation and
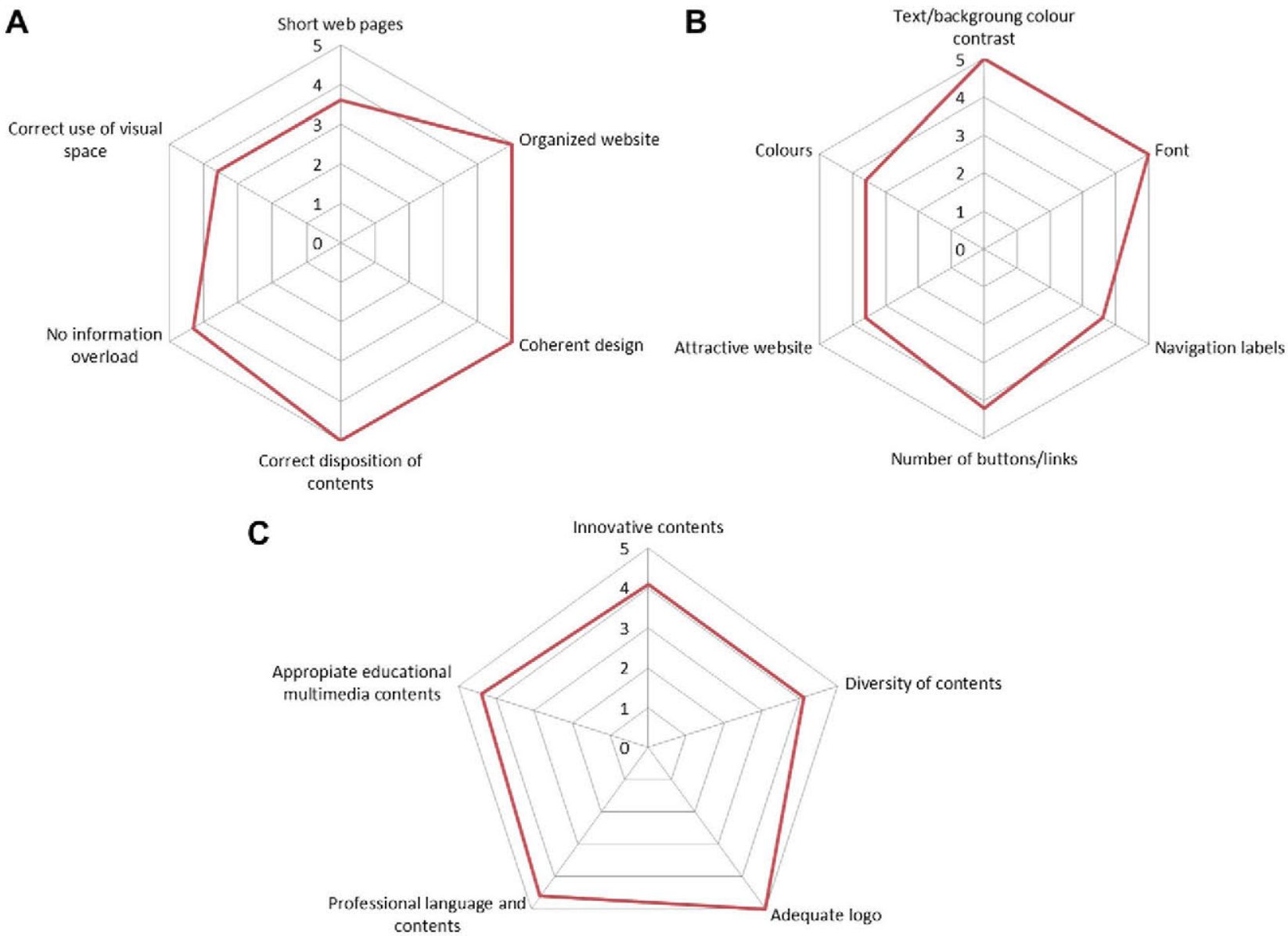

Fig. 4 - Preliminary TELMA environment validation results for usability: (A) layout; (B) design; (C) content. Bioengineering and Telemedicine Centre (GBT). 

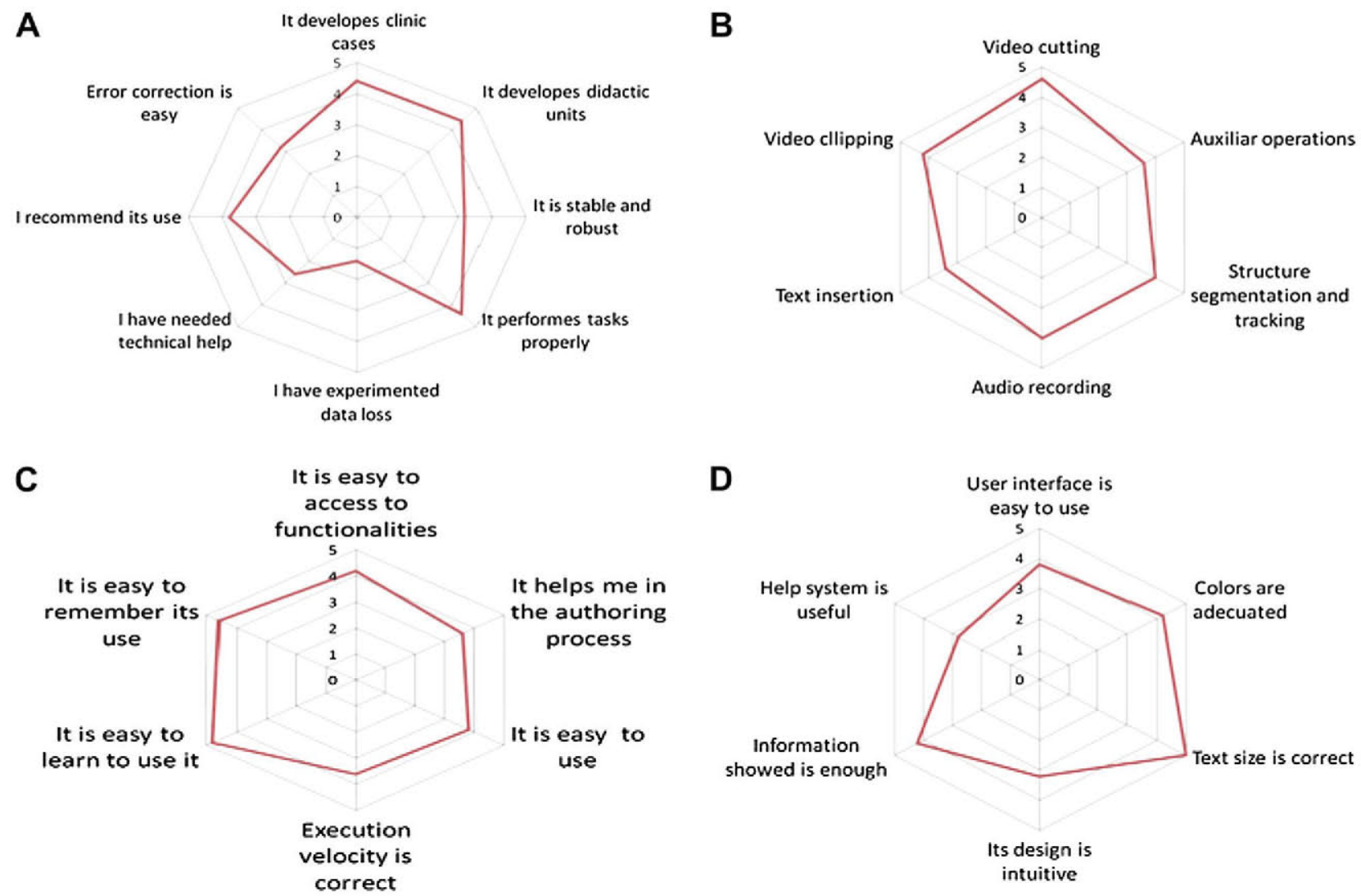

Fig. 5 - Preliminary authoring tool validation results: (A) operation of the tool; (B) video editor functionalities; (C) use and learning facility; (D) usability. Bioengineering and Telemedicine Centre (GBT).

tracking functionalities are suitable for training purposes. Thus, they allow focusing trainees' attention.

\section{Discussion}

We present a new TEL environment, the TELMA platform. TELMA enhances the user's experience using a four-pillared architecture: (1) an authoring tool; (2) a learning content and knowledge management system; (3) an evaluation module; and (4) a professional network for collaborative learning between users. A preliminary validation process of the environment and the multimedia authoring tool has shown its potential relevance in MIS cognitive skills training.

Conventional MIS cognitive skills training has been complemented by the launching of e-learning sites in surgical education, making for an interesting alternative of electronic and distance learning over traditional approaches [27]. Current training environments have many advantages, including ubiquity, continuous access, interactivity, and immediate feedback to users. They usually rely on the use of endoscopic videos to offer complete surgical libraries that can be accessed anytime and anywhere. However, they fail to provide formative paths for trainees to, based on their progress and interactions, guide them through their learning process; thus, they limit themselves in many cases to simple repositories of didactic content and videos. Moreover, any didactic material provided to the site must be preformatted by the author, as no authoring tool for content editing is provided. Finally, interactive collaboration for teamwork learning between users is not sufficiently exploited.

TELMA aims to overcome these limitations and take surgical e-learning technologies one step ahead based on its four-pillared structure. Surgical video information is presented as its multimedia didactic content core. Early familiarization with the view present in the operating rooms will shorten trainee learning curves. Moreover, video processing facilitates the enrichment of didactic information contained in the videos, enhancing their formative capacity.

Furthermore, multimedia content should have a high didactic quality. Due to the importance of the didactic content in the success of the environment, the development of an efficient and simple authoring tool is required. The proposed solution allows for the creation and editing of structured didactic content that can be uploaded or retrieved directly from the learning environment. Its simplicity in design makes it a user-friendly tool accessible to surgeons with different degrees of computer knowledge. In addition, it does not limit itself simply to the creation of didactic units or case reports, but includes a complete video editor that allows actions from simple enhancement operations to advanced segmentation and tracking features of elements within the video, as well as the possibility of creating associated clips. In the current implementation, the offline authoring tool connects 
seamlessly with the KMS and the environment, allowing for easy transfer of content. However, several technological challenges were encountered in the inclusion of the online authoring tool within the environment. Because of processing loads and connection issues, the current online version supports simple editing and clipping functions; thus, advanced processing features are left for the downloadable version.

The inclusion of a knowledge management system that allows for a personalized experience is one of the great technological challenges of the environment. For the smooth running of this module, data are required not only on the users' preferences, set manually during the registration process, but also on their performance and their interactions and networks within TELMA, as well as on other users' behaviors. These requirements lead to the partial solution of an issue commonly known as the "cold start problem," which happens especially in the first phases of deployment, when user data are too scarce to predict a complete behavioral model. The problem is addressed by means of an ontology-based system, which allows the creation of user profiles dynamically, based on similar behavior patterns among users.

One of the keys to success for any training program, whether for surgery or for any other field of knowledge, is the ability to provide immediate constructive feedback to the trainee during an exercise (formative) or after evaluation (summative). The evaluation module allows for this goal in two ways: (1) in a visible form to the user, in the form of evaluation and progress reports; and (2) under a hidden layer that interacts with the KMS and the recommendation system to construct the formative path of the user. Moreover, thanks to the editor included in the authoring tool, the deployment of evaluation units is easy and intuitive.

The professional network poses one of the most interesting dilemmas in the validation process, and there are several factors in play that will determine its final usefulness, including the surgeons' availability to interact with other users, their willingness to participate, and their degree of familiarity with web technologies, among other factors. Our initial hypothesis is that this module will gain in importance in the medium term, as surgeons and trainees who are adapted to web 2.0 technologies become more numerous.

The acceptance that an environment such as TELMA may gain among the surgical community is still unclear, and an exhaustive validation process should be carried out involving more users and with different surgical expertise. Other sites, such as WebSurg, have been able to mobilize a great number of users, which testifies to the usefulness of web technologies as a learning medium. Extensive clinical introduction will be a critical stage that can help improve and ensure the quality of the system. Throughout the design process, a close collaboration with clinical partners has guided the development of the environment. A simple but potent tool with a user-friendly interface is provided as per the requirements of an expert committee comprising both clinicians and software development experts. Navigation should be improved to increase the effectiveness as a conventional day-to-day education process, making the TELMA didactic process an enjoyable activity. Moreover, preliminary results have shown good acceptance of the functionalities for the creation and editing of didactic resources provided by the authoring tool, stressing its ease of use and its functional value, as well as guiding possible optimizations related to usability.

\section{Conclusions}

MIS cognitive skills training is a vital part of a surgeon's education. This work presents a collaborative technologyenhanced learning environment that will permit trainees to improve their knowledge and skills anytime and anywhere. TELMA comprises the following: (1) a multimedia didactic authoring tool; (2) a learning content and knowledge management system (LCKMS); (3) an objective evaluation module; and (4) a surgical professional network. Training with TELMA will be fully supported by automatic and immediate skills assessment, adaptive monitoring of the learning curve, and a professional network of surgeons with different experience to ensure a holistic and complete learning experience. TELMA's TEL environment is currently installed and being tested at the Jesús Usón Minimally Invasive Surgery Centre and at the Son Llàtzer Spanish hospital. Future improvements will deal with important issues such as multiplatform development. These improvements will address technical challenges, design frameworks, and development experiences that integrate multiple mobile devices (e.g., phones, tablet PCs). Nevertheless, it will be in the final validation processes and pilot programs within Spanish hospitals that the acceptance of TELMA will be fully established.

\section{Acknowledgment}

This research has been partially funded by the TELMA project (TSI-020110-2009-85) of the Spanish Ministry of Industry, Tourism and Trade. The TELMA project is led by ATOS Research \& Innovation with the Bioengineering and Telemedicine Centre from the Technical University of Madrid as scientific coordinator. The rest of the participants are CEPAL, Jesús Usón Minimally Invasive Surgery Centre, Son Llàtzer hospital, and ISID Media Asset Management.

\section{REFERENCES}

[1] Cuschieri A. Laparoscopic surgery: current status, issues and future developments. Surgeon 2005;3:125.

[2] Aggarwal R, Moorthy K, Darzi A. Laparoscopic skills training and assessment. Br J Surg 2004;91:1549.

[3] Halsted WS. The training of surgeons. Johns Hopkins Hosp Bull 1904;15:267.

[4] Dankelman J, Chmarra MK, Verdaasdonk EGG, Stassen LPS, Grimbergen CA. Fundamental aspects of learning minimally invasive surgical skills. Minim Invasive Ther Allied Technol 2005;14:247.

[5] Park AE, Witzke D. The surgical competence conundrum. Surg Endosc 2002;16:555.

[6] Moore RG, Loening S, Bishoff JT, Docimo SG, Bonnett R. Minimally invasive urologic surgery. Oxfordshire: Taylor \& Francis; 2005. 
[7] Rosser JC, Herman B, Risucci DA, Rosser L, Merrell RC. Effectiveness of a CD-ROM multimedia tutorial in transferring cognitive knowledge essential for laparoscopic skill training. Am J Surg 2000;179:320.

[8] Rosser JC, Murayama M, Gabriel NH. Minimally invasive surgical training solutions for the twenty-first century. Surg Clin N Am 2000;80:1607.

[9] Williamson RD, Smoak EP. Embracing edutainment with interactive e-learning tools. Journal of Extension 2005;43: 5IAW2.

[10] Chen K. Research on study-type instruction of college physics based on open source LCMS[J]. Journal of Xuzhou Education College; 2008.

[11] Fu-Chien K, Chih-Wei T, Zhi-Hua J. The design of embedded LCMS broker with load-balancing function. In: Proceeding of the Sixth International Conference on Machine Learning and Cybernetics. Hong Kong, 2007;7:3770.e6

[12] Sánchez-González P, Cano AM, Oropesa I, SánchezMargallo FM, del Pozo F, Gómez EJ. Laparoscopic video analysis for training and image guided surgery. Minim Invasive Ther Allied Technol 2011;20:311.

[13] Mutter D, Vix M, Dallemagne B, Perretta S, Leroy J, Marescaux J. WeBSurg: an innovative educational web site in minimally invasive surgery-principles and results. Surg Innov 2011;18:8.

[14] http://www.webop.de/; 2012 [accessed March 2012].

[15] http://www.surgytec.com/; 2012 [accessed March 2012].

[16] Sanchez-Peralta LF, Sanchez Margallo FM, Pagador Carrasco JB, et al. E-MIS: e-learning and multimedia contents for minimally invasive surgery. Minim Invasive Ther Allied Technol 2010;10:29.

[17] Sánchez-Margallo FM, Sánchez-Peralta LF, Pagador JB, et al. Nuevas tecnologías en cirugía de mínima invasión. Encuesta sobre e-learning y contenidos multimedia. Cirugía Española 2010;88:223.
[18] Romero-Zaldivar VA, Burgos D. Meta-Mender: a meta-rule based recommendation system for educational applications. Proc Comp Science 2010;1:2877.

[19] Wanzel KR, Ward M, Reznick RK. Teaching the surgical craft: from selection to certification. Curr Prob Surg 2002; 39:574.

[20] Oropesa I, Sánchez-González P, Cano AM, Lamata P, Sánchez-Margallo FM, Gómez EJ. Objective evaluation methodology for surgical motor skills assessment. Minim Invasive Ther Allied Technol 2010;10:55.

[21] Oropesa I, Sánchez-González P, Lamata P, et al. Methods and tools for objective assessment of psychomotor skills in laparoscopic surgery. J Surg Res 2011;171:e81.

[22] Cho H, Gayb G, Davidson B, Ingraffea A. Social networks, communication styles, and learning performance in a CSCL community. Comput Educ 2007;49:309.

[23] Harasim L, Hiltz SR, Teles L, Turoff M. Learning networks: a field guide to teaching and learning online. Cambridge: Cambridge University Press; 1995.

[24] Haythornthwaite C. Building social networks via computer networks: creating and sustaining distributed learning communities. In: Renninger KA, editor. Building virtual communities: learning and change in cyberspace. Pennsylvania: Swarthmore College; 2002. p. 159-90.

[25] Cho H, Stefanone M, Gay G. Social information sharing in a CSCL community. In: Proceedings of the Conference on Computer Support for Collaborative Learning: Foundations for a CSCL Community 2002. p. 43-50.

[26] Likert R. A technique for the measurement of attitudes. Arch Psychol 1932;140:1.

[27] Ortega Moran JF, Pagador JB, Sánchez Peralta LF, Gómez Aguilera EJ, Noguera Aguilar J, Sánchez Margallo FM. E-MIS validity applied to TELMA enhanced learning environment. 26th International Congress and Exhibition of Computer Assisted Radiology and Surgery, 2012, in press. 